\title{
Combined analysis of genome-wide expression and copy number profiles to identify key altered genomic regions in cancer
}

\author{
Celia Fontanillo", Sara Aibar ${ }^{1}$, Jose Manuel Sanchez-Santos², Javier De Las Rivas ${ }^{\text {* }}$ \\ From X-meeting 2011 - International Conference on the Brazilian Association for Bioinformatics and Compu- \\ tational Biology \\ Florianópolis, Brazil. 12-15 October 2011
}

\begin{abstract}
Background: Analysis of DNA copy number alterations and gene expression changes in human samples have been used to find potential target genes in complex diseases. Recent studies have combined these two types of data using different strategies, but focusing on finding gene-based relationships. However, it has been proposed that these data can be used to identify key genomic regions, which may enclose causal genes under the assumption that disease-associated gene expression changes are caused by genomic alterations.

Results: Following this proposal, we undertake a new integrative analysis of genome-wide expression and copy number datasets. The analysis is based on the combined location of both types of signals along the genome. Our approach takes into account the genomic location in the copy number (CN) analysis and also in the gene expression (GE) analysis. To achieve this we apply a segmentation algorithm to both types of data using paired samples. Then, we perform a correlation analysis and a frequency analysis of the gene loci in the segmented CN regions and the segmented GE regions; selecting in both cases the statistically significant loci. In this way, we find $\mathrm{CN}$ alterations that show strong correspondence with GE changes. We applied our method to a human dataset of 64 Glioblastoma Multiforme samples finding key loci and hotspots that correspond to major alterations previously described for this type of tumors.
\end{abstract}

Conclusions: Identification of key altered genomic loci constitutes a first step to find the genes that drive the alteration in a malignant state. These driver genes can be found in regions that show high correlation in copy number alterations and expression changes.

\section{Background}

Acquisition of somatic genetic alterations plays an important role in the development of cancer. Several systematic efforts have addressed the study of genetic alterations to characterize human cancers [1,2], including: copy-number alterations (CNAs), translocations, insertions or single-nucleotide polymorphisms (SNPs). Most of these approaches are focused on finding frequent alterations, which occur in a high number of cases.

\footnotetext{
* Correspondence: jrivas@usal.es

'Cancer Research Center (CIC-IBMCC), Consejo Superior de Investigaciones

Científicas (CSIC), Campus Miguel de Unamuno, Salamanca, Spain

Full list of author information is available at the end of the article
}

According to the selective pressure theory, a genomic alteration that confers an advantage to a malignant state is likely to be found in more tumors than expected by chance [3]. However, most methods that look for recurrent aberrations using copy number information find many regions, containing many genes $[4,5]$. Therefore, to identify recurrently altered genomic regions -biologically relevant- it is necessary to integrate gene and genome information, as proposed by Akavia et al. [3]. Several reports have recently shown that integrative strategies can be very useful to identify driver genes, considering the hypothesis that disease-associated gene expression changes are frequently induced by genomic alterations $[3,6-10]$.
C Biomed Central

(c) 2012 Fontanillo et al.; licensee BioMed Central Ltd. This is an open access article distributed under the terms of the Creative Commons Attribution License (http://creativecommons.org/licenses/by/2.0), which permits unrestricted use, distribution, and reproduction in any medium, provided the original work is properly cited. 
Most of these reports are focused on finding gene-based relationships.

Built on these hypotheses -that relate transcriptomic and genomic alterations-, we propose a new integrative method based on the location of both types of signals along the genome. Our method takes into account the genomic loci, both in the copy number $(\mathrm{CN})$ analysis and also in the gene expression (GE) analysis, and applies the segmentation step proposed by Ortiz-Estevez et al. [11]. These authors designed a method for robust comparison between $\mathrm{CN}$ and GE using paired samples. Such approach is based on a search for correlation between segmented $\mathrm{CN}$ regions and segmented GE regions to find the most significant simultaneous alterations. We follow this approach introducing two new steps to asses the matching between CN and GE loci: (i) first, a signal correlation analysis; (ii) second, an alteration frequency analysis. Using these analyses we propose a set of significantly altered genomic regions in the studied pathological state. In order to show the performance and demonstrate the value of our method, we use a dataset of 64 Glioblastoma Multiforme (GBM) samples with paired measurements of GE and $\mathrm{CN}$ (taken from $[7,8]$ ).

\section{Results and discussion}

The method is designed for combined analysis of datasets from two types of genome-wide arrays: DNA genomic microarrays and RNA expression microarrays. These arrays provide copy number and expression quantitative data, respectively. The analysis places both types of signals along the genome, taking into account the gene loci for the $\mathrm{CN}$ data and the GE data. The rationale of the method is to search for copy number alterations with a major influence in the expression levels of the genes encoded. As a distinctive element from other integrative approaches we do not consider only SNPs or genes individually. We take into account the gene loci following the strategy described in [11], that is based on the application of the same smoothing and segmentation algorithm to $\mathrm{CN}$ and GE in order to establish comparable regions. Once we get the smoothed segments, we perform two independent analyses for each gene loci: a signal correlation analysis and an alteration frequency analysis. (The workflow described in Materials and Methods, presented in last figure, illustrates the procedure of the method including these two independent analyses).

\section{Analysis of correlation between gene expression and copy number levels}

The method matches the $\mathrm{CN}$ and GE segmented signals within each chromosomal region -i.e., the $\log 2$ ratio signals of the corresponding segments- and selects the gene loci that show a significant correlation. These loci can be considered candidate hotspots. In Figure 1 we present the results of this analysis done for the GBM dataset, marking in purple the number of gene loci with Pearson Correlation Coefficient $r \geq 0.60$ (that corresponds to a Bonferroni-adjusted p-value $<0.005)$. Such cutoff $(r \geq 0.60)$ includes around $55 \%$ of the human gene loci, providing a good coverage with a significant p-value. Setting more stringent cutoffs reduces the coverage too much: $r \geq 0.70$ includes only $\sim 26 \%$ of the gene loci; $r \geq 0.80$ includes only $\sim 6 \%$.

The number of probes in the SNP arrays -used to calculate the segmented signals for $\mathrm{CN}$ - is large and uniform along the genome. However, in the expression arrays some genomic regions do not have enough allocated gene loci and the number of probes is sparse. This fact is a problem when a GE segment includes outliers (i.e. gene locus which have expression levels very different from the mean of their neighbours). To solve this problem, we look for statistically significant outliers within the GE segments -which were at least in $1 / 3$ of the samples- and we recalculate the signal correlation between their unsegmented GE and the corresponding $\mathrm{CN}$ segments. In this way, we find a new set of gene loci with correlation $r \geq 0.60$, which is added to the initial set of candidate hotspots identified. This step of the procedure is important to recover some gene loci with quite significant correlation (e.g. EGFR or SEC61G), which were missed in the first step due to the described problem.

\section{Analysis of frequencies for the categorical states Up-Gain and Down-Loss}

The method also proposes to find the genomic regions that present a significant GE and $\mathrm{CN}$ alteration in the same direction. To assess this, we included a second selective step based on stratification of the segmented data. The genomic regions are stratified in several categories: up-regulation (U), down-regulation (D) or no-change (N) for expression; and gain $(\mathbf{G})$, loss $(\mathbf{L})$ or no-change $(\mathbf{N})$ for copy number. This approach allows a discretization of the genomic regions into 9 different categories as shown in Figure 2 (inserted table): U-G, N-G, D-G, U-N, N-N, D-N, U-L, N-L, D-L. Figure 2 also presents the empirical cumulative distributions for these 9 categories of the GBM samples per gene loci, counting the frequency of samples for all the gene loci in each category. As expected, the distributions show that the "no change" (i.e. N-N, neutralneutral) is the most frequent state. The analysis of distributions also finds some regions that show a clear correspondence between $\mathrm{GE}$ and $\mathrm{CN}$ alterations: i.e. the scenario where GE up-regulation is observed co-located with a $\mathrm{CN}$ gain (U-G category) and the scenario where GE down-regulation is co-located with a CN loss (D-L category). Our interest focuses on these regions, since they are the ones altered in the same way in both types of data. The analysis of the empirical frequency distributions for 


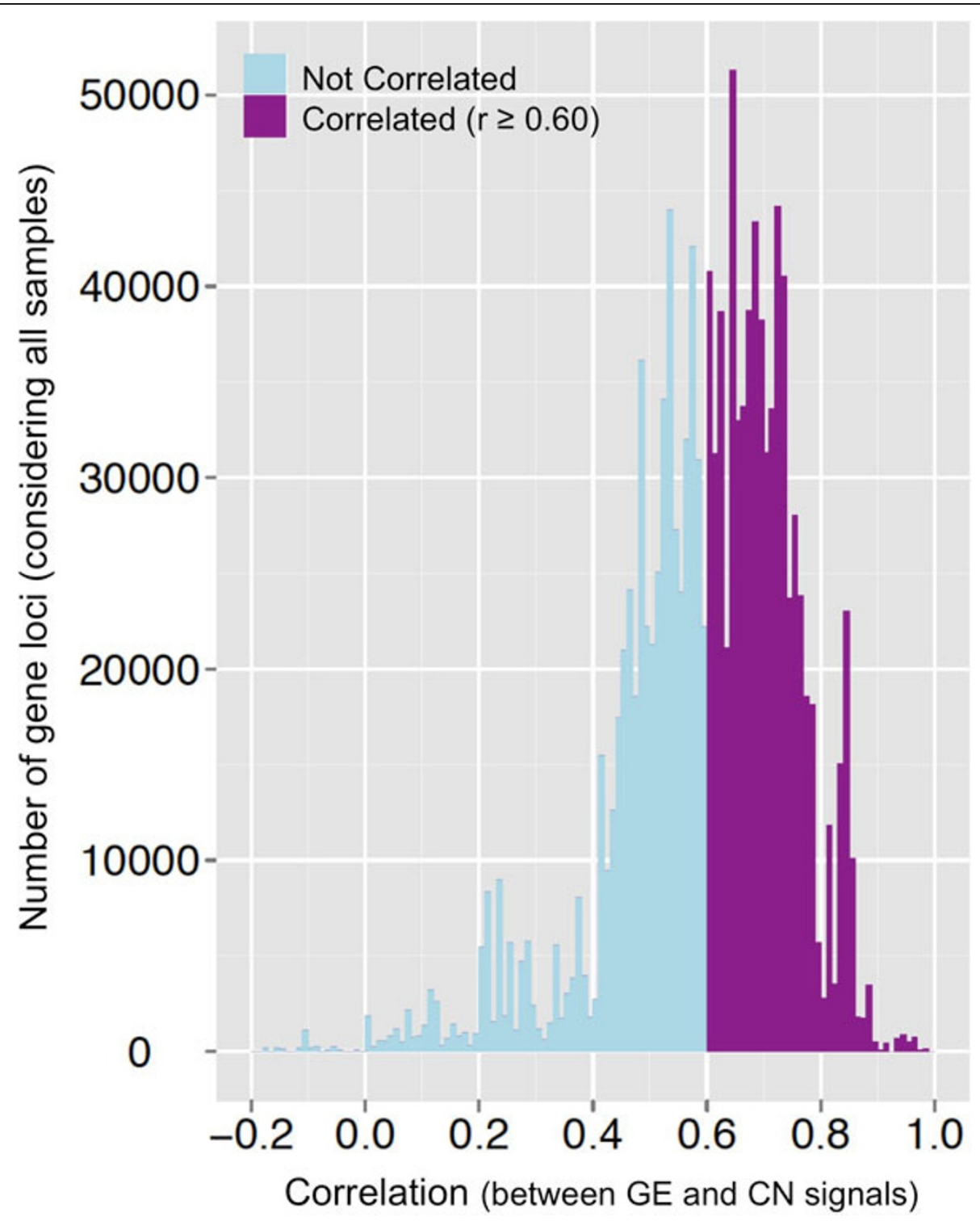

Figure 1 Density distribution of the correlation coefficients between GE and CN for the GBM dataset. Purple represents the number of gene loci that present significant correlation $(r \geq 0.60)$ between gene expression and copy number signals, counted considering all the samples. Blue are the rest, not considered significant.

the $\mathbf{U}-\mathbf{G}$ and $\mathbf{D}-\mathbf{L}$ categories allows identifying the frequency cutoffs that correspond to the $10 \%$ upper quantiles. These cutoffs were: 13 samples for $\mathbf{U}-\mathbf{G}$ and 11 samples for D-L (out of 64 in GBM dataset). The set up of these thresholds identifies those genomic regions that are the most frequently assigned to such altered categories (U-G and D-L) in the studied dataset.

\section{Genome-wide identification of hotspots: candidate key genomic regions}

Our method identifies candidate key regions that show high correlation between $\mathrm{CN}$ and GE and that are frequently altered in the same direction, in both types of signals. The overlapping between the regions with the most significant correlation and the ones with the highest frequencies of simultaneous alteration ( $\mathrm{CN}$ and $\mathrm{GE}$ ) along the genome, will constitute hotspots where putative driver genes are likely to be encoded.

Figure 3 presents the combined view of GE and $\mathrm{CN}$ alterations on the complete genome obtained for the GBM dataset. The graph shows the alteration frequency, either in $\mathrm{CN}$ or in GE independently, along all the genome (22 human chromosomes). The dark colors correspond to GE up-regulated regions (red) or down-regulated regions (green), and the light colors -placed on top-correspond to $\mathrm{CN}$ gains (pale red) and losses (pale green). These results 




show that the method finds the alterations previously described for $\mathrm{CN}$ in GBM cancer [12,13]. In fact, the most frequent alterations in glioblastoma are the gain of chromosome 7 and the loss of chromosome 10. Our analysis finds such alterations in $\mathrm{CN}$, and also finds their correlation with GE up-regulation for chromosome 7 and with GE down-regulation for chromosome 10. Figure 4 presents a detailed view of the alterations that occur in chromosome 7. It includes a profile of the regions with significant correlation (purple dots along the chromosome) and a profile of the frequency of $\mathbf{U}-\mathbf{G}$ regions (pale red). They cover nearly the complete chromosome. A figure with the representation for all the 22 human chromosomes for the GBM samples is included as Additional File 3.

Key genomic regions found for the 64 paired GBM cancer samples

As shown in Figure 3, the method presented in this work allows the identification of relevant altered genomic regions suffering significant changes in most of the GBM samples. The results also show that many of the detected $\mathrm{CN}$ alterations and GE changes overlap along the genome. These regions can be proposed as relevant "hotspots". In Table 1 and Table 2 we present a detailed description of the common genomic regions found in GBM; indicating the correlation and frequency of the $\mathbf{U}-\mathbf{G}$ regions (Table 1 , which includes 19 regions), and the $\mathbf{D}-\mathbf{L}$ regions (Table 2, which includes 24 regions). The tables include the correlation between GE and $\mathrm{CN}$ for each region (as average correlation for all the gene loci); and the percentage of samples -frequency-in each region, counting only the samples where simultaneous GE and $\mathrm{CN}$ alterations occur: either up gene expression and gain in copy number $(\mathbf{U}-\mathbf{G})$ or down gene expression and loss in copy number (D-L). The regions detected are in the chromosomes that suffer the most significant changes in GBM samples: U-G, chr 7 and chr 20; D-L chr 10, chr 13, chr 14 and chr 22. The tables also include the genes enclosed in these regions. The most remarkable changes correspond to a 




Figure 3 Combined view of GE and CN alterations obtained for the GBM dataset. The regions for all the human chromosomes (chr) that are altered either in $\mathrm{CN}$ or in GE are presented along the whole genome keeping the proportional size of the chromosomes. The graph shows the frequency of such alterations in the GBM samples. The colors correspond to GE up-regulated regions (in red) or down-regulated regions (in green), and -plotted on top- the CN gains (in pale red) or the CN losses (in pale green). Blue lines mark the regions that change in more than $25 \%$ of the GBM patients. The chromosomes with most significant changes (that present large regions included in the categories $\mathbf{U}-\mathbf{G}$ or $\mathbf{D}-\mathbf{L}$ ) are labeled: U-G chr 7, chr 19, chr 20; D-L chr 10, chr 13, chr 22.

large part of chr 7 (U-G) and to a large part of chr 10 (D-L). Two important genes are precisely located in these chromosomes: EGFR (in chr 7) usually up-regulated and PTEN (in chr 10) usually down-regulated $[12,13]$. PTEN is not found in our analysis, but it has been reported an absence of PTEN alterations in more than half of de novo glioblastomas and more than $90 \%$ of glioblastomas developed from a pre-existing lower grade gliomas [14], which has been linked to the presence of additional tumor suppressor genes on chr 10, such as LGI1 [15] and MXI1 [16]. We found these two genes in regions 8 and 10 of the D-L list (Table 2), and we observed a very variable profile of PTEN in the GBM samples. These facts may indicate that PTEN is not the best genomic marker for this altered

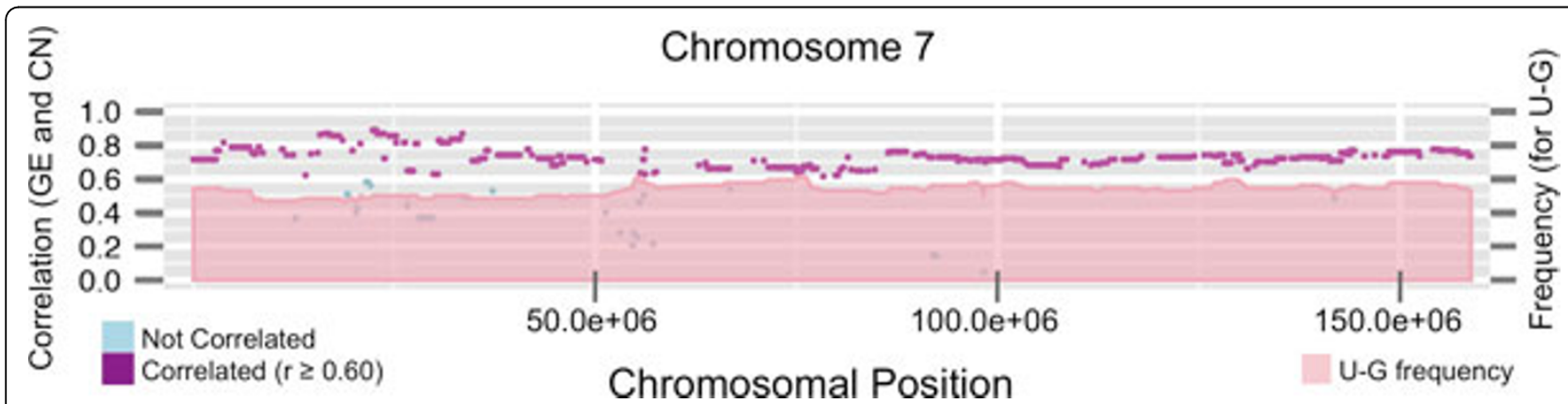

Figure 4 Detailed view of chromosome 7 showing the CN and GE correlation and the U-G category frequency for the GBM dataset The genomic regions for chromosome 7 are represented in X-axis. Blue and purple dots show the correlation coefficients between $\mathrm{CN}$ and $\mathrm{GE}$ for each gene loci (purple when $r \geq 0.60$ ). Pink profile represents the frequency values for the Up-Gain category (U-G). 
Table 1 Significant U-G regions with the associated genes.

\begin{tabular}{|c|c|c|c|c|c|c|c|c|}
\hline regions & chr & cytobands & start & end & Correlation (average $\mathrm{r}$ coefficient) & U-G Frequency (average \%) & Number of genes & gene symbols \\
\hline 1 & 7 & p22.3,p22.2,p22.1,... & 13912 & 12407180 & 0.75 & 53.13 & 97 & PDGFA,PRKAR1B,HEATR2,... \\
\hline 2 & 7 & p21.2,p21.1 & 13980952 & 18581782 & 0.83 & 48.34 & 16 & ETV1,DGKB,TMEM195,... \\
\hline 3 & 7 & p21.1 & 19741898 & 19786077 & 0.77 & 48.44 & 2 & TWISTNB,TMEM196 \\
\hline 4 & 7 & p21.1 & 20735744 & 20825207 & 0.81 & 50.00 & 2 & $\mathrm{ABCB} 5, \mathrm{SP} 8$ \\
\hline 5 & 7 & p15.3,p15.2 & 22277336 & 26372745 & 0.85 & 50.00 & 28 & RAPGEF5,L6,TOMM7,... \\
\hline 6 & 7 & p15.2 & 26682190 & 27829944 & 0.68 & 50.00 & 18 & SKAP2,HOXA1,HOXA2,... \\
\hline 7 & 7 & p14.3 & 29901392 & 33407268 & 0.78 & 50.00 & 29 & WIPF3,SCRN1,FKBP14,... \\
\hline 8 & 7 & $p 14.3, p 14.2$ & 34692740 & 36658380 & 0.72 & 48.44 & 13 & NPSR1,DPY19L1,TBX20,... \\
\hline 9 & 7 & $\mathrm{p} 14.1, \mathrm{p} 13, \mathrm{p} 12.3, \ldots$ & 37856706 & 50759460 & 0.72 & 49.60 & 83 & GPR141,TXNDC3,SFRP4,... \\
\hline 10 & 7 & p11.2 & 54819940 & 54826939 & $0.77 *$ & 59.38 & 1 & SEC61G \\
\hline 11 & 7 & p11.2 & 55086725 & 55275031 & $0.77 *$ & 60.94 & 1 & EGFR \\
\hline 12 & 7 & p11.2 & 55572215 & 56043680 & 0.70 & 59.06 & 5 & VOPP1,SEPT14,ZNF713,... \\
\hline 13 & 7 & p11.2 & 56125502 & 56171766 & 0.70 & 57.81 & 4 & CCT6A,SUMF2,PHKG1,... \\
\hline 14 & 7 & $\mathrm{p} 11.2, \mathrm{p} 11.1, \mathrm{q} 11.21$ & 57269897 & 66582330 & 0.66 & 56.56 & 25 & ERV3,VKORC1L1,GUSB,... \\
\hline 15 & 7 & $q 11.22, q 11.23, q 21.11, \ldots$ & 69660980 & 91851882 & 0.68 & 57.74 & 131 & AUTS2,WBSCR17,CALN1,... \\
\hline 16 & 7 & q21.2,q21.3, & 92738082 & 97975672 & 0.72 & 56.51 & 36 & SAMD9,SAMD9L,HEPACAM2,... \\
\hline 17 & 7 & $\mathrm{q} 22.1, \mathrm{q} 22.3, \mathrm{q} 31.1, \ldots$ & 98456252 & 141707080 & 0.71 & 55.94 & 343 & TMEM130,TRRAP,SMURF1,... \\
\hline 18 & 7 & $\mathrm{q} 34, \mathrm{q} 35, \mathrm{q} 36.1, \ldots$ & 141954920 & 158879258 & 0.75 & 56.26 & 154 & TRBV12-2,TRBC1,PRSS1,... \\
\hline 19 & 20 & $\mathrm{p} 13, \mathrm{p} 12.3, \mathrm{p} 12.2, \ldots$ & 72762 & 62897316 & 0.83 & 25.62 & 570 & DEFB125,DEFB126,DEFB12,... \\
\hline
\end{tabular}

Table with the significant Up-regulated and Gained (U-G) regions, indicating: chromosomal bands covered by each region; percentage of samples (\%) that are in the U-G category in each region (calculated as average frequency for all the gene loci in the region); correlation between GE and CN for each region (calculated as average correlation of the gene loci in the region); number of genes located in each region. Tota number of GBM samples analysed: $\mathrm{N}=64$. Marked with * the correlations calculated between unsegmented GE and segmented $\mathrm{CN}$. Due to size limitations the table only includes a maximum of 3 cytobands or 3 genes. Complete information corresponding to this U-G regions is included as supplementary material: Additional-file-1. 
Table 2 Significant D-L regions with the associated genes.

\begin{tabular}{|c|c|c|c|c|c|c|c|c|}
\hline regions & chr & cytobands & start & end & Correlation (average $\mathrm{r}$ coefficient) & U-G Frequency (average \%) & Number of genes & gene symbols \\
\hline 1 & 10 & p14 & 9365826 & 11653762 & 0.62 & 57.19 & 5 & CUGBP2,C10orf31,USP6NL,... \\
\hline 2 & 10 & $\mathrm{p} 13, \mathrm{p} 12.33, \mathrm{p} 12.31, \ldots$ & 16746068 & 24410224 & 0.64 & 59.98 & 39 & RSU1,CUBN,TRDMT1,... \\
\hline 3 & 10 & $\mathrm{p} 12.1, \mathrm{p} 11.23, \mathrm{p} 11.22, \ldots$ & 25189544 & 47921720 & 0.63 & 60.50 & 103 & PRTFDC1,ENKUR,THNSL1,... \\
\hline 4 & 10 & p11.2 & 38238795 & 38265453 & $0.60 *$ & 60.94 & 1 & ZNF25 \\
\hline 5 & 10 & $q 11.22_{2 \prime} q 11.23$ & 49203737 & 53404614 & 0.68 & 62.91 & 42 & FAM25C,BMS1P7,PTPN20C,... \\
\hline 6 & 10 & $\mathrm{q} 21.1, \mathrm{q} 21.2, \mathrm{q} 21.3, \ldots$ & 59989486 & 82351987 & 0.65 & 64.07 & 152 & IPMK,CISD1,UBE2D1,... \\
\hline 7 & 10 & q23.1, & 84190870 & 87742781 & 0.66 & 65.63 & 9 & NRG3,GHITM,PCDH21,... \\
\hline 8 & 10 & $q 23.31, q 23.32, q 23.33$ & 91498034 & 97024055 & 0.62 & 67.19 & 39 & KIF20B,HTR7,RPP30,... \\
\hline 9 & 10 & q24.1 & 97391080 & 97763109 & 0.62 & 66.41 & 6 & ALDH18A1,TCTN3,ENTPD $1, \ldots$. \\
\hline 10 & 10 & $\mathrm{q} 24.1, \mathrm{q} 24.2, \mathrm{q} 24.31, \ldots$ & 98081203 & 134474152 & 0.67 & 68.70 & 253 & DNTT,OPALIN,TLL2,... \\
\hline 11 & 13 & $q 12.13, q 12.2$ & 27693163 & 28017254 & 0.60 & 28.13 & 5 & USP12,RPL21,RASL11A, $\ldots$ \\
\hline 12 & 13 & $q 12.2_{\prime \prime} q 12.3$ & 28367434 & 30381664 & 0.61 & 30.29 & 13 & GSX1,PDX1,ATP5EP2,... \\
\hline 13 & 13 & $q 13.3, q 14.11, q 14.12, \ldots$ & 35881808 & 61059301 & 0.73 & 35.48 & 124 & NBEA,MAB21L1,DCLK1,... \\
\hline 14 & 13 & $\mathrm{q} 21.32, \mathrm{q} 21.33$ & 67340716 & 70478658 & 0.62 & 35.94 & 2 & PCDH9,KLHL1 \\
\hline 15 & 13 & $q 22.2, q 22.3, q 31.1$ & 76451567 & 80912598 & 0.63 & 37.40 & 15 & KCTD12,IRG1,CLN5,... \\
\hline 16 & 13 & q31.3, & 92785210 & 94467454 & 0.62 & 34.38 & 2 & GPC5,GPC6 \\
\hline 17 & 13 & $\mathrm{q} 32.1, \mathrm{q} 32.2, \mathrm{q} 32.3, \ldots$ & 95812885 & 106130800 & 0.64 & 33.35 & 38 & ABCC4,CLDN10,DZIP1,... \\
\hline 18 & 13 & q33.3 & 108170700 & 108931486 & 0.95 & 27.73 & 4 & FAM155A,LIG4,ABHD13,... \\
\hline 19 & 13 & q34 & 110422550 & 111549152 & 0.89 & 23.44 & 9 & IRS2,COL4A1,COL4A2, ... \\
\hline 20 & 14 & $\mathrm{q} 11.2, \mathrm{q} 12, \mathrm{q} 13.1, \ldots$ & 19686156 & 70583360 & 0.75 & 18.44 & 389 & TTC5,CCNB1IP1,PARP2,... \\
\hline 21 & 14 & $\mathrm{q} 24.2$ & 71478110 & 72101080 & 0.74 & 17.19 & 2 & PCNX,SIPA1L1 \\
\hline 22 & 14 & $\mathrm{q} 24.2_{2 \prime} \mathrm{q} 24.3$ & 73223406 & 76274521 & 0.69 & 17.19 & 52 & DPF3,DCAF4,ZFYVE1,... \\
\hline 23 & 14 & $q 24.3 „ q 31.1$ & 77825772 & 80673424 & 0.75 & 17.19 & 14 & TMED8,AHSA1,ISM2,... \\
\hline 24 & 22 & $q 11.1, q 11.21, q 11.22, \ldots$ & 16157622 & 51224902 & 0.73 & 24.93 & 490 & POTEH,CESK1,XKR3,... \\
\hline
\end{tabular}

Table with the significant Down-regulated and Lost (D-L) regions, indicating: chromosomal bands covered by each region; percentage of samples (\%) that are in the D-L category in each region (calculated as average frequency for all the gene loci in the region); correlation between GE and CN for each region (calculated as average correlation of the gene loci in the region); number of genes located in each region. Total number of GBM samples analysed: $\mathrm{N}=64$. Marked with * : correlations calculated between unsegmented GE and segmented CN. Due to size limitations the table only includes a maximum of 3 cytobands or 3 genes. Complete information corresponding to this D-L regions is included as supplementary material: Additional-file- 2 . 
region. By contrast, we found RB1 tumor suppressor in region 13 of the $\mathbf{D}$-L list; and this gene -included in chr 13- is a clear candidate to drive the alteration of tumor cells. With respect to EGFR, it has the highest $\mathbf{U}-\mathbf{G}$ frequency observed $(60.9 \%$, Table 1$)$ and therefore the method reveals this gene locus as the most common GE up-regulated and $\mathrm{CN}$ gained in the GBM samples. The alteration of EGFR can be associated with other genes that regulate its function, also found by the method. This is the case of VOPP1 and RAB11FIP2. VOPP1 is also known as ECOP (EGFR-coamplified and overexpressed protein) or GASP (Glioblastoma-amplified secreted protein), and is found in region 12 of the $\mathbf{U}-\mathbf{G}$ list (Table 1). RAB11FIP2 is a suppressor of the endocytic internalization of EGFR and it is found in region 10 of the D-L list (Table 2) [17]. The presence of these genes in the hotspots found for GBM supports the value of the method described. There are many other interesting genes in the identified altered genomic regions, that can be useful for further investigations on the disease studied.

Complete information corresponding to the genes found in the significant $\mathbf{U}-\mathbf{G}$ regions and $\mathbf{D}-\mathbf{L}$ regions is included respectively as supplementary material in Additional-file-1 (for the data corresponding to Table 1) and Additional-file-2 (for the data corresponding to Table 2).

\section{Conclusions}

The combined analysis of $\mathrm{CN}$ and GE data obtained using DNA genome and RNA expression microarrays for paired samples is a very powerful approach to uncover key altered regions in a biological state studied. We present a robust method to find genomic regions that show simultaneous significant changes in both $\mathrm{CN}$ and GE. Our calculations applied to a cancer dataset find expected known genomic alterations and many others identified as key altered genomic regions. This approach is also proposed as an adequate strategy to identify driver or causal genes under the hypothesis that disease-associated gene expression changes are frequently induced by genomic alterations.

\section{Materials and methods \\ Data}

In this study we use a dataset of 64 human samples from Glioblastoma Multiforme (GBM) [7] that includes for each sample: Affymetrix DNA microarrays applied to detect of genome-wide $\mathrm{CN}$ changes and Affymetrix RNA expression microarrays applied to detect of GE changes. We used the same subgroup of samples that was previously analysed in Ortiz-Estevez et al. [11].

\section{GE and CN normalization and signals calculation}

GE data were processed using RMA algorithm [18] applied to the human gene expression microarrays: Affymetrix HGU133 plus 2.0 (using the same strategy followed in $[19,20])$. CRMAv2 algorithm [21] was applied to normalize the raw data and obtain the signals from the Affymetrix Human Mapping 500K SNP arrays. The processed signals were divided by the median of the normal samples for each element (SNP or gene) and then the $\log 2$ was computed. These $\log 2$ ratio signals were smoothed and segmented using Circular Binary Segmentation (CBS) algorithm [22] with the default parameters implemented in the DNAcopy $R$ package.

\section{Correlation between GE and CN}

Pearson Correlation Coefficients ( $r$ ) of the segmented GE and $\mathrm{CN}$ data were calculated taking the values of the segmented copy number and gene expression at the central point of the genomic position for each gene. P-values for the correlation coefficient of every gene loci were computed and adjusted by Bonferroni method. The established threshold for the selection of significantly correlated gene loci was correlation coefficient $r \geq 0.60$, which corresponds to adjusted $\mathrm{p}$-value $<0.005$. When using the gene loci GE unsegmented signal, the same correlation threshold and p-value cutoff were applied.

\section{Frequency of U-G and D-L alterations}

The thresholds that define DNA copy number gains and losses and up and down gene regulation were established applying $\mathrm{k}-$ Means algorithm, fixing three clusters $(\mathrm{k}=3)$ on the segmented data, and done independently for the $\mathrm{CN}$ data and for the GE data. The $\mathrm{CN}$ data values were classified into gained $(\mathbf{G})$, lost $(\mathbf{L})$ or no-change $(\mathbf{N})$ and the GE values were classified as up-regulated (U), downregulated (D) or no-change $(\mathbf{N})$. The thresholds found by k-Means for $\mathrm{CN}$ in the GBM dataset were $>0.19$ (of the $\log 2$ ratio signals) for gain and $<-0.15$ for loss. The thresholds found for GE in the GBM were $>0.10$ (of the log2 ratio signals) for up-regulation and $<-0.12$ for down-regulation. A contingency table with the 9 possible categorical states for the two types of data was built for every gene locus. A cutoff threshold was set up for the frequency of up-regulated and gained (U-G) and for the down-regulated and lost (D-L) categories, based on the empirical cumulative distributions of the categories. Taking into account the gene loci, the significant altered regions were defined as the ones that had a frequency $\geq$ than the upper $10 \%$ quantile of the distribution of $\mathbf{U}-\mathbf{G}$ or the distribution of D-L.

\section{General workflow for identification of key regions in the genome}

Following the steps described above, we present a general workflow (Figure 5) that illustrates the strategy to achieve a combined paired analysis of datasets from genome-wide microarrays, both for $\mathrm{GE}$ and $\mathrm{CN}$. 


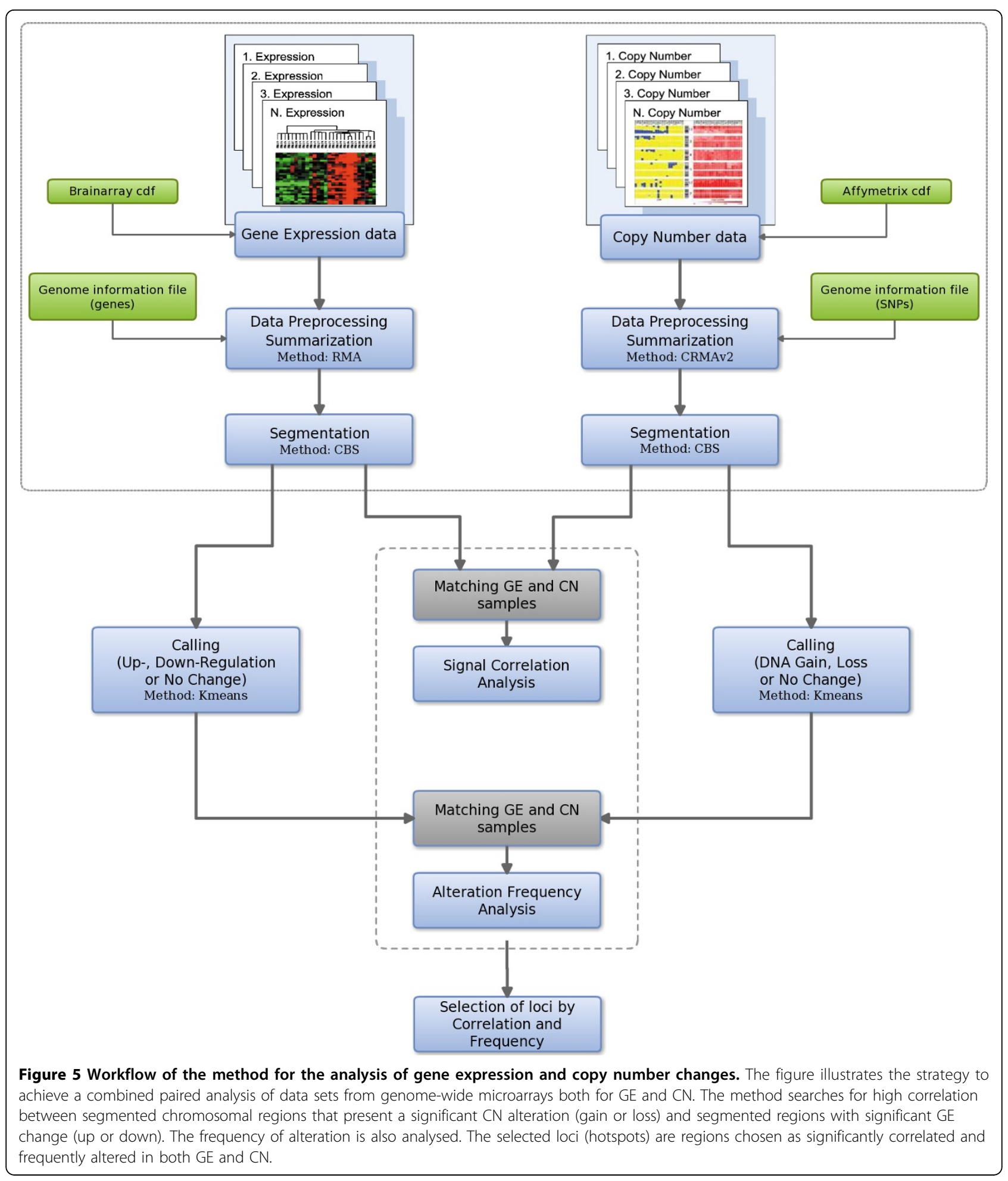

The workflow includes the different steps, the applied methods and the progression of the analysis. The strategy designed searches for high correlation between chromosomal regions that present a significant $\mathrm{CN}$ alteration (as gain or loss) and regions with significant GE change (as up or down). In this way, it determines which $\mathrm{CN}$ alterations have a strong influence on GE patterns. Key regions, i.e. hotspots in the genome, are defined as those regions simultaneously chosen as significantly correlated and frequently altered in both $\mathrm{GE}$ and $\mathrm{CN}$. 


\section{Additional material}

\section{Additional file 1: Spreadsheet with the complete data} corresponding to Table 1.

Additional file 2: Spreadsheet with the complete data corresponding to Table 2 .

Additional file 3: Detailed view of all the 22 chromosomes showing the $\mathrm{CN}$ and GE correlation and the U-G or D-L categories frequency for the GBM dataset. The genomic regions are represented in $X$-axis. Blue and purple dots show the correlation coefficients between $\mathrm{CN}$ and $G E$ for each gene loci (purple when $r \geq 0.60$ ). Pink and green profiles represent the frequency values for the Up-Gain (U-G) category or the Down-Loss (D-L) category respectively.

\section{Acknowledgements}

This work has been supported by funds provided by the Local Government Junta de Castilla y León (JCyL, ref. project: CSI07A09), by the Spanish Government (ISCiii, ref. project PS09/00843) and by the European Commission (Research Grant ref. FP7-HEALTH-2007-223411). SA thanks the $J C Y L$ and the European Social Fund (ESF-EU) for a research grant. The funders had no role in study design, data collection and analysis, decision to publish, or preparation of the manuscript.

This article has been published as part of BMC Genomics Volume 13 Supplement 5, 2012: Proceedings of the International Conference of the Brazilian Association for Bioinformatics and Computational Biology (X-meeting 2011). The full contents of the supplement are available online at http://www.biomedcentral.com/bmcgenomics/supplements/13/S5.

\section{Author details}

${ }^{1}$ Cancer Research Center (CIC-IBMCC), Consejo Superior de Investigaciones Científicas (CSIC), Campus Miguel de Unamuno, Salamanca, Spain.

${ }^{2}$ Department of Statistics, University of Salamanca (USAL), Salamanca, Spain.

\section{Authors' contributions}

CF carried out most of the analyses, developed the proposed method and drafted the manuscript. SA helped in the computational analyses and in the presentation of the results. JMSS participated in the design of the study and in the statistical methods applied. JDLR conceived of the study, participated in its design and coordination and wrote the main manuscript. All authors read and approved the final manuscript.

\section{Competing interests}

The authors declare that they have no competing interests.

Published: 19 October 2012

\section{References}

1. Futreal PA, Coin L, Marshall M, Down T, Hubbard T, Wooster R, Rahman N, Stratton MR: A census of human cancer genes. Nature Rev Cancer 2004, 4:177-83.

2. Stratton MR, Campbell PJ, Futreal PA: The cancer genome. Nature 2009, 458:719-24.

3. Akavia UD, Litvin O, Kim J, Sanchez-Garcia F, Kotliar D, Causton HC, Pochanard P, Mozes E, Garraway L a, Pe'er D: An integrated approach to uncover drivers of cancer. Cell 2010, 143:1005-17.

4. Beroukhim R, Getz G, Nghiemphu L, Barretina J, Hsueh T, Linhart D, Vivanco I, Lee JC, Huang JH, Alexander S, Du J, Kau T, Thomas RK, Shah K, Soto H, Perner S, Prensner J, Debiasi RM, Demichelis F, Hatton C, Rubin MA, Garraway LA, Nelson SF, Liau L, Mischel PS, Cloughesy TF, Meyerson M, Golub TA, Lander ES, Mellinghoff IK, Sellers WR: Assessing the significance of chromosomal aberrations in cancer: methodology and application to glioma. Proc Nat Acad Sci USA 2007, 104:20007-12.

5. Beroukhim R, Mermel CH, Porter D, Wei G, Raychaudhuri S, Donovan J, Barretina J, Boehm JS, Dobson J, Urashima M, Mc Henry KT, Pinchback RM, Ligon AH, Cho Y-J, Haery L, Greulich H, Reich M, Winckler W, Lawrence MS, Weir BA, Tanaka KE, Chiang DY, Bass AJ, Loo A, Hoffman C, Prensner J, Liefeld T, Gao Q, Yecies D, Signoretti S, Maher E, Kaye FJ, Sasaki H,
Tepper JE, Fletcher JA, Tabernero J, Baselga J, Tsao M-S, Demichelis F, Rubin MA, Janne PA, Daly MJ, Nucera C, Levine RL, Ebert BL, Gabriel S, Rustgi AK, Antonescu CR, Ladanyi M, Letai A, Garraway LA, Loda M, Beer DG, True LD, Okamoto A, Pomeroy SL, Singer S, Golub TR, Lander ES, Getz G, Sellers WR, Meyerson M: The landscape of somatic copy-number alteration across human cancers. Nature 2010, 463:899-905.

6. Pollack JR, Sørlie T, Perou CM, Rees CA, Jeffrey SS, Lonning PE, Tibshirani R, Botstein D, Børresen-Dale A-L, Brown PO: Microarray analysis reveals a major direct role of DNA copy number alteration in the transcriptional program of human breast tumors. Proc Nat Acad Sci USA 2002, 99:12963-8.

7. Kotliarov Y, Steed ME, Christopher N, Walling J, Su Q, Center A, Heiss J, Rosenblum M, Mikkelsen T, Zenklusen JC, Fine HA: High-resolution global genomic survey of 178 gliomas reveals novel regions of copy number alteration and allelic imbalances. Cancer Res 2006, 66:9428-36.

8. Kotliarov Y, Kotliarova S, Charong N, Li A, Walling J, Aquilanti E, Ahn S, Steed ME, Su Q, Center A, Zenklusen JC, Fine $\mathrm{H}$ a: Correlation analysis between single-nucleotide polymorphism and expression arrays in gliomas identifies potentially relevant target genes. Cancer Res 2009, 69:1596-603.

9. Turner N, Lambros MB, Horlings HM, Pearson A, Sharpe R, Natrajan R, Geyer FC, van Kouwenhove M, Kreike B, Mackay A, Ashworth A, van de Vijver MJ, Reis-Filho JS: Integrative molecular profiling of triple negative breast cancers identifies amplicon drivers and potential therapeutic targets. Oncogene 2010, 29:2013-23.

10. Kim Y-A, Wuchty S, Przytycka TM: Identifying causal genes and dysregulated pathways in complex diseases. PLoS Computational Biology 2011, 7:e1001095.

11. Ortiz-Estevez M, De Las Rivas J, Fontanillo C, Rubio A: Segmentation of genomic and transcriptomic microarrays data reveals major correlation between DNA copy number aberrations and gene-loci expression. Genomics 2011, 97:86-93.

12. De Tayrac M, Etcheverry A, Aubry M, Saïkali S, Hamlat A, Quillien V, Le Treut A, Galibert MD, Mosser J: Integrative genome-wide analysis reveals a robust genomic glioblastoma signature associated with copy number driving changes in gene expression. Genes Chromosomes Cancer 2009, 48:55-68.

13. Ruano Y, Mollejo M, Ribalta T, Fiaño C, Camacho Fl, Gómez E, de Lope AR, Hernández-Moneo JL, Martínez P, Meléndez B: Identification of novel candidate target genes in amplicons of glioblastoma multiforme tumors detected by expression and CGH microarray profiling. Molecular Cancer 2006, 5:39.

14. Reifenberger G, Collins VP: Pathology and genetics of astrocytic gliomas. J Mol Med 2004, 82:656-670.

15. Chernova OB, Somerville RP, Cowell JK: A novel gene, LGI1, from 10q24 is rearranged and downregulated in malignant brain tumors. Oncogene 1998, 17:2873-2881.

16. Wechsler DS, Shelly CA, Petroff CA, Dang CV: MXI1, a putative tumor suppressor gene, suppresses growth of human glioblastoma cells. Cancer Res 1997, 57:4905-4912.

17. Cullis DN, Philip B, Baleja JD, Feig LA: Rab11-FIP2, an adaptor protein connecting cellular components involved in internalization and recycling of epidermal growth factor receptors. J Biol Chem 2002, 277:49158-49166.

18. Irizarry R a, Hobbs B, Collin F, Beazer-Barclay YD, Antonellis KJ, Scherf U, Speed TP: Exploration, normalization, and summaries of high density oligonucleotide array probe level data. Biostatistics 2003, 4:249-64.

19. Vicent S, Luis-Ravelo D, Antón I, García-Tuñón I, Borrás-Cuesta F, Dotor J, De Las Rivas J, Lecanda F: A novel lung cancer signature mediates metastatic bone colonization by a dual mechanism. Cancer Res 2008, 68:2275-85.

20. Hernández JA, Rodríguez AE, González M, Benito R, Fontanillo $C$, Sandoval V, Romero M, Martín-Núñez G, de Coca AG, Fisac R, Galende J, Recio I, Ortuño F, García JL, De Las Rivas J, Gutiérrez NC, San Miguel JF, Hernández JM: A high number of losses in 13q14 chromosome band is associated with a worse outcome and biological differences in patients with B-cell chronic lymphoid leukemia. Haematologica 2009, 94:364-371.

21. Bengtsson H, Wirapati P, Speed TP: A single-array preprocessing method for estimating full-resolution raw copy numbers from all Affymetrix genotyping arrays including GenomeWideSNP 5 \& 6. Bioinformatics 2009, 25:2149-56. 
22. Venkatraman $E S$, Olshen $A B$ : A faster circular binary segmentation algorithm for the analysis of array CGH data. Bioinformatics 2007, 23:657-63.

doi:10.1186/1471-2164-13-S5-S5

Cite this article as: Fontanillo et al: Combined analysis of genome-wide expression and copy number profiles to identify key altered genomic regions in cancer. BMC Genomics 2012 13(Suppl 5):S5.

Submit your next manuscript to BioMed Central and take full advantage of:

- Convenient online submission

- Thorough peer review

- No space constraints or color figure charges

- Immediate publication on acceptance

- Inclusion in PubMed, CAS, Scopus and Google Scholar

- Research which is freely available for redistribution

Submit your manuscript at www.biomedcentral.com/submit

() BioMed Central 